\title{
BEAM DYNAMICS OPTIMIZATION FOR THE XFEL PHOTO INJECTOR
}

\author{
MIKHAIL KRASILNIKOV \\ PITZ, Deutsches Elektronen-Synchrotron, \\ Platanenallee 6, Zeuthen, 15738, Germany \\ mikhail.krasilnikov@desy.de
}

The main challenge for the European XFEL photo injector is the production of $1 \mathrm{nC}$ electron beams with a normalized transverse emittance of $0.9 \mathrm{~mm}$ mrad. The photo injector setup consists of a 1.5-cell L-band rf gun cavity supplied with solenoids for beam focusing and emittance compensation and the first accelerating section with 8 TESLA superconducting cavities. The first 4 cavities are used as a booster to provide by proper choice of its position, gradient and phase matching conditions for the emittance conservation.

For optimization of the beam dynamics in the photo injector, a staged algorithm, based on ASTRA simulations, has been developed. The first stage considers the emission of electrons from a photo cathode. The cathode laser energy and its transverse parameters are adjusted to produce a bunch charge of $1 \mathrm{nC}$ in presence of space charge forces (including image charge at the cathode) and Schottky-like effects. The second stage contains rf gun cavity and solenoid optimization. The booster position, gradient and initial phase are optimized at the third stage yielding the minimum emittance at the photo injector exit. Results of the XFEL photo injector optimization will be presented.

Besides simulations experimental studies towards XFEL photo injector are carried out. The photo injector test facility at DESY in Zeuthen (PITZ) develops photo injectors for FELs, including FLASH and the European XFEL. A thorough comparison of measured data with results of beam dynamics simulations is one of the main PITZ goals. Detailed experimental studies on photo emission processes, thermal emittance, transverse and longitudinal phase space of the electron beam are being performed together with beam dynamics simulations. This aims to result in better understanding of beam dynamics in high brightness photo injectors. Experimentally obtained photo injector characteristics (like thermal emittance) have to be used in an additional optimization of the photo injector resulting in more realistic beam dynamics simulations. Results of these studies will be reported as well.

Keywords: Photo injector; FEL; emittance.

PACS numbers: 29.27.Bd, 29.25.Bx

\section{Introduction}

Modern linac based Free-Electron Lasers (FELs) require high brightness electron beams. rf photo injectors are capable of producing high charge electron beams with extremely small transverse emittance. A transverse normalized beam emittance of 
$0.9 \mathrm{~mm}$ mrad for $1 \mathrm{nC}$ bunch charge at the injector exit is the main challenge for the European XFEL photo injector. ${ }^{1}$

\section{XFEL Photo Injector Concept}

High densities of the transverse phase space (or small emittance) imply space charge dominated beams in a photo injector. Since the high charge is extracted from the photo cathode at almost zero energy the Coulomb repulsion forces play a significant role in the dynamics of the electrons within the bunch. Strong nonlinearities of the space charge field in the cathode vicinity result in beam emittance growth. In order to provide a small emittance in the photo injector, several techniques have to be applied. Solenoid compensation ${ }^{2}$ implies reversing the correlations along the bunch by applying a solenoid lens. By proper choice of the solenoid parameters the space charge force acts at some distance reducing transverse phase space nonlinear correlations along the bunch and resulting in a projected emittance minimum. Electron beams accelerated in the rf gun still remain space charge dominated, which leads to an emittance increase. In order to keep the emittance small further beam acceleration by a booster must be applied. For efficient emittance conservation, matching conditions based on the invariant envelope technique provide a quasi laminar beam regime. ${ }^{3}$ This requires proper choice of the booster position, gradient and phase.

The XFEL photo injector consists of an rf gun, a standard accelerating module (ACC1), and a diagnostic section. The electron bunch is emitted from a $\mathrm{Cs}_{2} \mathrm{Te}$ cathode illuminated by a UV laser pulse in a high accelerating field $(60 \mathrm{MV} / \mathrm{m}$ peak field on the cathode) and accelerated up to $6.3 \mathrm{MeV}$ in the normal conducting $1.3 \mathrm{GHz}$ 1,5-cell rf gun cavity. A solenoid, centered $0.276 \mathrm{~m}$ downstream of the cathode, focuses the beam into the first accelerator module which is located after a drift length of about $3.5 \mathrm{~m}$. A bucking solenoid is to be used to compensate the magnetic field at the cathode. The accelerator module increases the energy to about 160-170 MeV.

Cathode laser pulse shaping is a key issue for the photo injector performance. In order to reduce the space charge effect, especially during emission from the cathode a flat top temporal profile with 20 ps FWHM and 2 ps rise and fall time is foreseen for the European XFEL photo injector. A radially homogeneous transverse profile is required with a diameter chosen as a compromise between thermal and space charge induced emittance.

\section{Simulation of Beam Dynamics in Photo Injector}

In order to optimize photo injector parameters, a number of beam dynamics simulations have been performed. The ASTRA code ${ }^{4}$ was used to simulate electron beam properties from the photo cathode till the photo injector exit $(z=15 \mathrm{~m})$. An electron beam has been tracked in the external electromagnetic fields of the rf gun with solenoids and the accelerating module (ACC1) with eight TESLA cavities. The first four cavities have been used as a booster, so the gradient and the phase 
of these cavities were tuned to minimize the beam emittance. RF gradient of the second four cavities was assumed close to the maximum available (peak field of $50 \mathrm{MV} / \mathrm{m}$ ), its phase has been tuned to minimize an rms energy spread of the electron beam.

\section{XFEL Photo Injector Optimization Strategy}

A multi-staged approach has been used for the optimization of the photo injector:

(1) emission from the photo cathode

(2) optimization of the rf gun parameters

(3) booster optimization

(4) minimization of the beam energy spread

The beam normalized rms projected emittance $\varepsilon=\sqrt{\varepsilon_{n x} \varepsilon_{n y}}$ has been used as a goal function for the minimization of the nominal XFEL photo injector setup:

$$
\Phi_{\text {goal }}=\left[\left.\varepsilon\right|_{z=15 m}+\left.L \cdot \frac{d \varepsilon}{d z}\right|_{z=15 m}\right] \cdot \Pi_{1} \cdot \Pi_{1} \ldots \cdot \Pi_{K},
$$

where not only the emittance but also its slope $\frac{d \varepsilon}{d z}$ is used to calculate the emittance trend at some distance $L$ (typically $L \sim 5 \mathrm{~m}$ ) from the injector exit $(z=15 \mathrm{~m})$. A penalty factor $\Pi_{n}=\Pi\left(p_{n}\right)$ has been used in order to eliminate solutions which can not be realized experimentally. This factor implies an exponential growth of the goal function if the photo injector parameter $p_{n}$ is outside the specified range $\left[p_{\text {from }} ; p_{\text {to }}\right]$ :

$$
\Pi(p)= \begin{cases}\exp \left(\frac{p_{\text {from }}-p}{p_{\text {to }}-p_{\text {from }}}\right), & \text { if } p<p_{\text {from }} \\ \exp \left(\frac{p-p_{\text {to }}}{p_{\text {to }}-p_{\text {from }}}\right), & \text { if } p>p_{\text {to }} \\ 1, & \text { otherwise }\end{cases}
$$

Various machine parameters and electron beam properties are considered for the penalty factor at different optimization stages. Mainly a longitudinal positions of the gun solenoid and the booster were affecting the penalty factor in the optimization of the XFEL photo injector.

The aperture of the FLASH/PITZ beam line (which is assumed to be similar to the XFEL one) has been also used; possible charge losses are considered as an additional contribution to the penalty factor.

\subsection{Emission from the photo cathode}

This step serves as a pre-run of the rf gun optimization (subsection 4.2). For the given cathode laser and rf gun parameters the laser power is tuned to produce a bunch charge of $1 \mathrm{nC}$. The tracking stops at $z=7 \mathrm{~cm}$ (right after the first iris of the gun cavity), what corresponds to the total extracted charge which will be 
transported downstream. Several ASTRA computations run in parallel to reduce computation time.

The main physical effects governing the emission process are space charge, including image charge from the cathode, and electromagnetic forces of the rf gun cavity. Another important emission feature is a Schottky-like effect ${ }^{5}$ in $\mathrm{rf} \mathrm{guns}$, i.e. the enhancement of the photo emission resulting from an electric field at the cathode surface. In other words, a bunch charge of $1 \mathrm{nC}$ could be reached in different ways. The simplest model is a "pure" photo effect, which implies no influence of the electric field at the cathode on the QE of the photo cathode. This does not exclude an integral impact of the space charge, especially in the case of extremely high space charge density at the cathode (e.g. small transverse size of the laser spot). Another model allows the emission due to the presence of an electric field at the moment of emission of the corresponding part of an electron beam. Both models are implemented in ASTRA. In the case of emission with Schottky-like effect the charge of macro particles $Q_{m p}$ is calculated according to: ${ }^{4}$

$$
Q_{m p}=\frac{Q_{b 0}+s_{1} \cdot E(t)+s_{2} \sqrt{E(t)}}{N_{p}},
$$

where $Q_{b 0}$ corresponds to the charge produced by photo effect ( $Q_{b 0} \sim$ laser power), the term $s_{1} \cdot E(t)+s_{2} \sqrt{E(t)}$ is related to the additional charge due to the Schottkylike effect in the presence of field $E(t)$ at a moment of the macro particle emission, $N_{p}$ is the number of macro particles used for simulations. This model is valid for both above mentioned emission models, $s_{1,2}=0$ corresponds to the "pure" photo effect.

Simulated rf gun phase scans for a given bunch charge are shown in Fig. 1. Two sets of simulations have been performed. The first did not apply Schottky-like effect, $Q_{b 0}=1.02 \mathrm{nC}$ in order to produce a $1 \mathrm{nC}$ beam at the $\mathrm{rf}$ gun launch phase of maximum energy gain (zero phase). The second simulation involves the macro particle charge dependence on the electric field at the cathode at the moment of emission according to Eq. (3) with $Q_{b 0}=0.5 \mathrm{nC}, s_{1}=0.16 \mathrm{nC} \cdot \mathrm{m} / \mathrm{MV}, s_{2}=$ $0.089 \mathrm{nC} \cdot(\mathrm{m} / \mathrm{MV})^{1 / 2}$.

Despite the charge and the beam energy for both simulations are the same, the transverse phase space differs significantly (Fig. 2) resulting in different transverse rms emittances at $z=4.3^{\mathrm{a}}: \varepsilon_{n, x}^{n o S c h o t t k y}=4.9 \mathrm{~mm} \cdot \mathrm{mrad}$ and $\varepsilon_{n, x}^{\text {Schottky }}=$ $2.5 \mathrm{~mm} \cdot \mathrm{mrad}$. These simulations were performed to illustrate the importance of the emission process in an rf gun and the necessity to take into account experimental data for correct simulations - namely, the gun phase scan for the given bunch charge, when Schottky-like effects are observed for $\mathrm{rf}$ guns with $C s_{2} T e$ photo cathodes. ${ }^{6}$ Usually experimental phase scans for a bunch charge are used to obtain constants $s_{1}$ and $s_{2}$ used in ASTRA simulations by fit of the simulated charge to the measured one. 


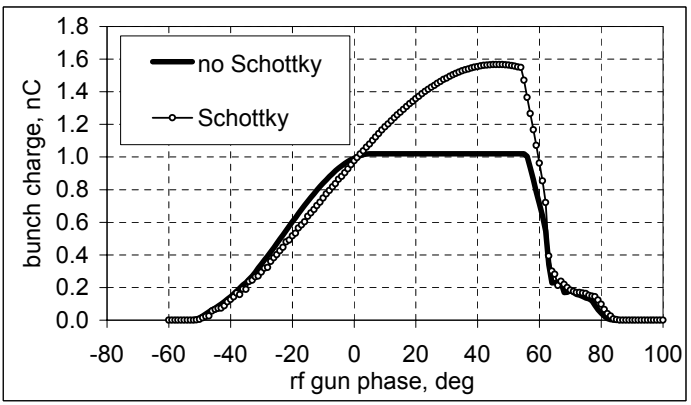

Fig. 1. Gun phase scan-bunch charge vs. gun launch phase. Zero phase is referred to as an rf gun launch phase yielding the maximum beam energy gain. Both ASTRA simulations were aimed to produce a $1 \mathrm{nC}$ electron beam at zero phase.
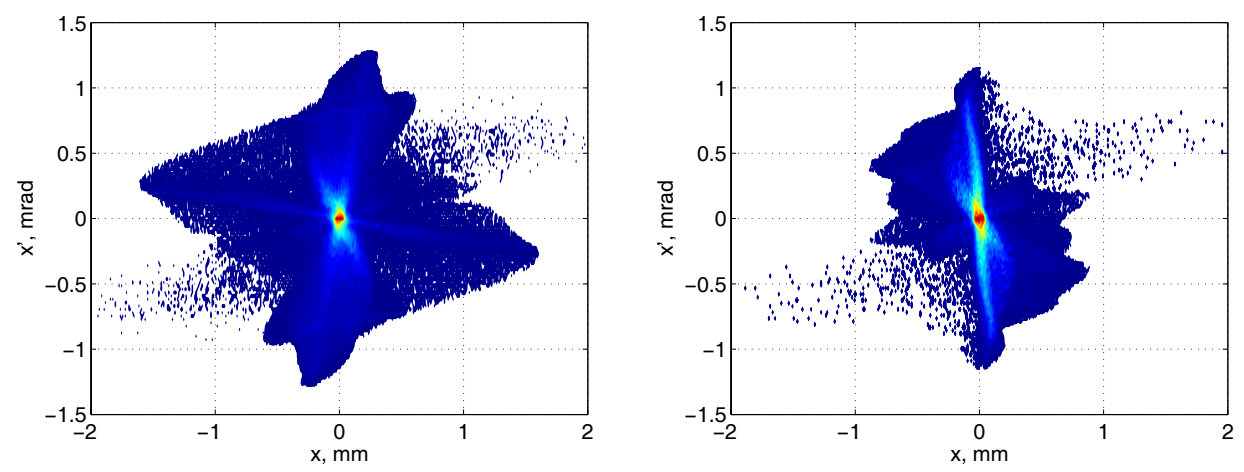

Fig. 2. Simulated transverse phase for the case without (left) and with Schottky-like effect (right).

\subsection{Optimization of the rf gun parameters}

Main parameters optimized at this stage are:

- cathode laser transverse rms size ${ }^{b}$

- main solenoid peak field

- longitudinal position of the main solenoid

- launch phase of the rf gun

The gun rf gradient has been fixed at $60 \mathrm{MV} / \mathrm{m}$ peak field at the cathode, which is the XFEL photo injector project parameter. An emittance reduction with an increase of the gun gradient ${ }^{7}$ has been taken into account. Further increase of the gun gradient is restricted by the limits of the $\mathrm{rf}$ system. ${ }^{8}$ 
The temporal laser profile was fixed to be a flat-top with 20 ps FWHM and 2 ps rise and fall time. The influence of the rise and fall time on the beam projected emittance has been studied additionally (see section 5.3). The pulse length is a compromise between space charge reduction during emission and nonlinearities in the longitudinal phase space due to the rf field of the gun and ACC1 cavities. These nonlinearities are planned to be compensated by the $3 \mathrm{rd}$ harmonics cavity ${ }^{1}$ operated strongly off-crest. The 3rd harmonics section is located in the linac downstream the injector and out of the scope of this paper.

The main contribution to the penalty factor at this stage is the longitudinal position of the main solenoid. Its further shift downstream the beam line is restricted by the mechanical construction of the coaxial coupler of the gun. This results in the solenoid centering at $z=0.276 \mathrm{~m}$, what is close to its actual position at PITZ and FLASH.

Definitely, an optimized transverse size of the cathode laser is also determined by the initial thermal emittance of the electron beam. According to the model of $C s_{2}$ Te cathodes, ${ }^{9}$ the initial kinetic energy of the photo electrons is $E_{k}=0.55 \mathrm{eV}$. This model does not include bending of energy levels in $C S_{2} T e$ in presence of electric field (rf and space charge) applied during electron emission from the cathode. Recent thermal emittance measurements at PITZ ${ }^{10}$ yielded $E_{k}=1.4 \mathrm{eV}$. Simulations for different values of the thermal kinetic energies have been performed (for results see section 5.3). The increase of the initial kinetic energy of electrons $E_{k}$ can be also (at least partially) explained by cathode surface imperfections (roughness, contamination level, etc).

The optimization of the rf gun parameters starts with tuning of the photo emission (see subsection 4.1). Afterward a $1 \mathrm{nC}$ electron beam is tracked until the beam waist (typically first $5 \mathrm{~m}$ of the beam line were sufficient to contain a beam waist). A beam monitor (containing all particle coordinates) near the waist location is saved and used as an input beam for the booster optimization (subsection 4.3)

In order to establish an initial simplex of rf gun parameters a Monte-Carlo ${ }^{11}$ method has been used. The best solutions have been used as initial simplexes for the subsequent optimization using the Nelder-Mead algorithm. ${ }^{12}$ Every optimization parameter has been centered to the initial guess and normalized to the range where significant emittance growth takes place. This range can be obtained by numerical study of the corresponding parameter influence on the emittance growth (see section 5.3). Typically 10\% emittance growth has been used in order to find search parameter ranges, needed for dimensionless variables used in the optimization algorithm.

\subsection{Booster optimization}

For each set of rf gun parameters the booster has to be optimized for a minimum emittance at the injector exit. ASTRA tracks the electron beam obtained from the previous stage (4.2). Main parameters to be optimized at this stage: 
- longitudinal position of the booster

- booster gradient (peak field)

- rf phase of the booster.

As it has been mentioned before the first four TESLA cavities of ACC1 are used as a booster for the XFEL photo injector. For the nominal photo injector settings gradient and phases in all the cavities of the booster were assumed to be equal.

The technique of the invariant envelope is used to obtain an initial guess for the booster optimization. The booster entrance is positioned near the electron beam waist, the booster normalized accelerating gradient $\gamma^{\prime}$ is chosen to provide a quasi laminar beam regime: ${ }^{3}$

$$
\gamma^{\prime}=\frac{2}{\sigma_{i}} \sqrt{\frac{I}{3 I_{0} \gamma_{i}}},
$$

where $\sigma_{i}$ and $\gamma_{i}$ are transverse rms size and normalized beam energy at the entrance of the booster, $I$ is a beam current, $I_{0}=17 \mathrm{kA}$ is the Alfven current. Such a proper choice of the initial parameters accelerates the 3-dimensional search procedure.

As contributions to the penalty factor the two following assumption have been used at this stage of optimization: 1) longitudinal start position of the booster has to be greater than $z=2.5 \mathrm{~m}$ due to mechanical restrictions of the beam line; 2 ) upper limit for the booster peak field was chosen to be $50 \mathrm{MV} / \mathrm{m}$ (close to the limit of the TESLA cavities capability).

\subsection{Minimization of the beam energy spread}

The second half of ACC1 consists of four TESLA cavities operated at $50 \mathrm{MV} / \mathrm{m}$ peak field. Their phases were synchronously tuned to minimize the rms energy spread of the beam at $z=15 \mathrm{~m}$.

Because of the weak dependence of the beam emittance on the phase of the second half of $\operatorname{ACC} 1\left(\phi_{3}\right)$ (Fig. 3), the phase with minimum rms momentum spread is chosen in order to conduct the beam into the matching section (minimizing chromatic effects). The phase of the absolute emittance minimum (emittance decrease $0.25 \%$ ) corresponds to rather large energy spread and this can cause a significant emittance growth in quadrupoles of the matching section.

\section{Optimized XFEL Photo Injector}

Beam dynamics optimization of the XFEL photo injector resulted in a setup summarized in Table $1^{\mathrm{c}}$.

Corresponding external electromagnetic fields along the beam line are shown in Fig. 4. 


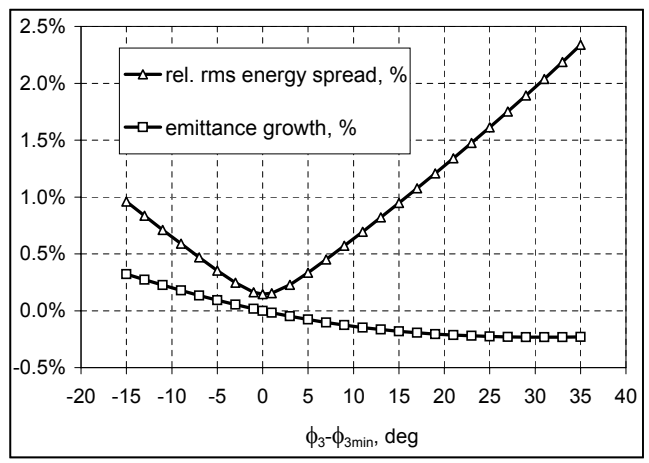

Fig. 3. Typical dependence of electron beam parameters on the phase $\phi_{3}$ of the second half of ACC1. Relative rms energy spread $\left(\sigma_{E} /\langle E\rangle\right)$ is plotted together with emittance growth $\varepsilon\left(\phi_{3}\right) / \varepsilon(0)-1$. Zero phase $\varepsilon_{3}=0^{\circ}$ corresponds here to the phase of the minimum energy spread.

Table 1. Optimized parameters of the XFEL photo injector.

\begin{tabular}{|c|c|c|c|}
\hline subsystem & parameter & value & unit \\
\hline rf gun cavity & $E_{\text {cath }}$ & 60 & $\mathrm{MV} / \mathrm{m}$ \\
\cline { 2 - 4 } & launch phase $\phi_{1 \text { opt }}$ & $\mathbf{- 0 . 7}$ & $\mathrm{deg}$ \\
\hline cathode laser & FWHM & 20 & $\mathrm{ps}$ \\
\cline { 2 - 4 } & rise/fall time & 2 & $\mathrm{ps}$ \\
\cline { 2 - 4 } & $\sigma_{x, y}$ & $\mathbf{0 . 4 5}$ & $\mathrm{mm}$ \\
\hline solenoid & peak field $B_{z}^{\text {opt }}$ & $\mathbf{- 0 . 2 2 9 4}$ & $\mathrm{T}$ \\
\cline { 2 - 4 } & center & $\mathbf{0 . 2 7 6}$ & $\mathrm{m}$ \\
\hline ACC1, cav.1-4 & cav.1 center & $\mathbf{4 . 0 4}$ & $\mathrm{m}$ \\
\cline { 2 - 4 } & peak field $E_{z}$ & $\mathbf{2 4 . 5}$ & $\mathrm{MV} / \mathrm{m}$ \\
\cline { 2 - 4 } & phase $\phi_{2 o p t}$ & $\mathbf{- 1 5}$ & $\mathrm{deg}$ \\
\hline ACC1, cav.5-8 & peak field $E_{z}$ & 50 & $\mathrm{MV} / \mathrm{m}$ \\
\cline { 2 - 4 } & phase & $\mathbf{5}$ & $\mathrm{deg}$ \\
\hline electron beam & charge, $\mathrm{Q}$ & 1 & $\mathrm{nC}$ \\
\cline { 2 - 4 } & thermal kinetic energy $E_{k}$ & 0.55 & $\mathrm{eV}$ \\
\cline { 2 - 4 } & mean energy & 167 & $\mathrm{MeV}$ \\
\cline { 2 - 4 } & rms energy spread & 0.25 & $\mathrm{MeV}$ \\
\cline { 2 - 4 } & $\begin{array}{c}\text { rms projected } \\
\text { normalized emittance } \\
\varepsilon_{x, y}(z=15 m)\end{array}$ & 0.7 & $\mathrm{~mm} \mathrm{mrad}$ \\
& & & \\
\hline
\end{tabular}

\subsection{Electron beam properties along the beam line}

Beam rms transverse size $\sqrt{\sigma_{x} \cdot \sigma_{y}}$ and rms projected transverse emittance $\sqrt{\varepsilon_{x} \cdot \varepsilon_{y}}$ are shown in Fig. 5. The transverse rms beam size of $240 \mu \mathrm{m}$ at the injector exit corresponds to the beta-function value of $\beta_{x, y}(z=15 \mathrm{~m})=28 \mathrm{~m}$.

Mean kinetic energy and rms energy spread are shown in Fig. 6, a relative energy spread of $0.15 \%$ has been simulated. 


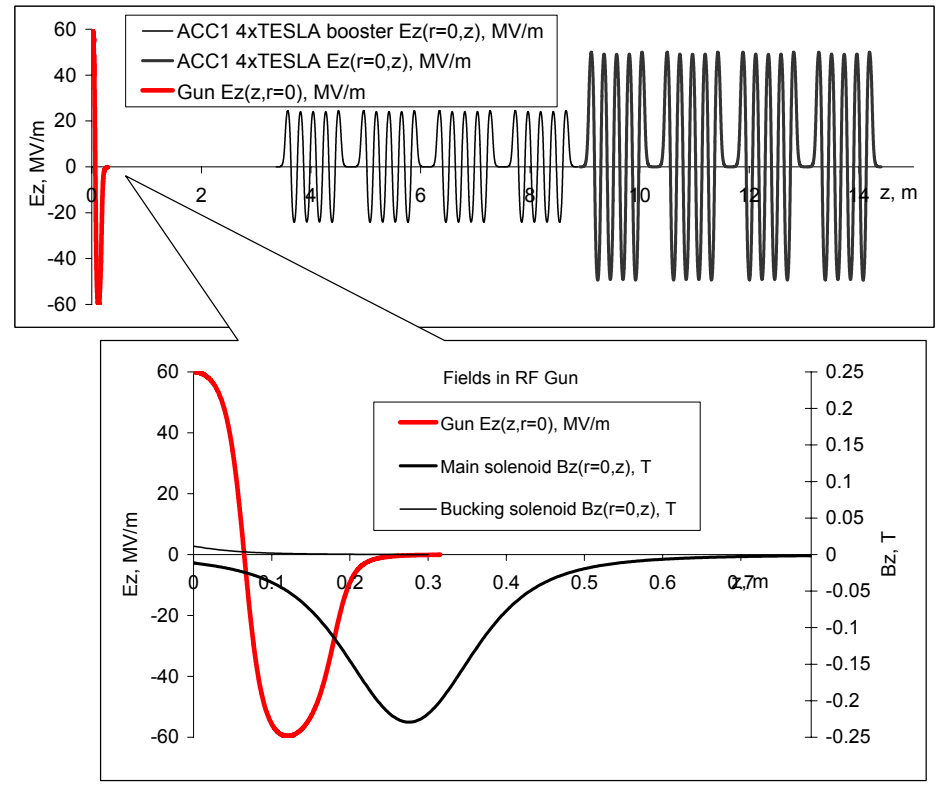

Fig. 4. Electromagnetic fields in the XFEL photo injector

RMS bunch length and longitudinal emittance are shown in Fig. 7 as a function of the distance from the cathode. An electron bunch length of $2 \mathrm{~mm}$ rms or $7 \mathrm{ps}$ FWHM has been obtained at the injector exit.

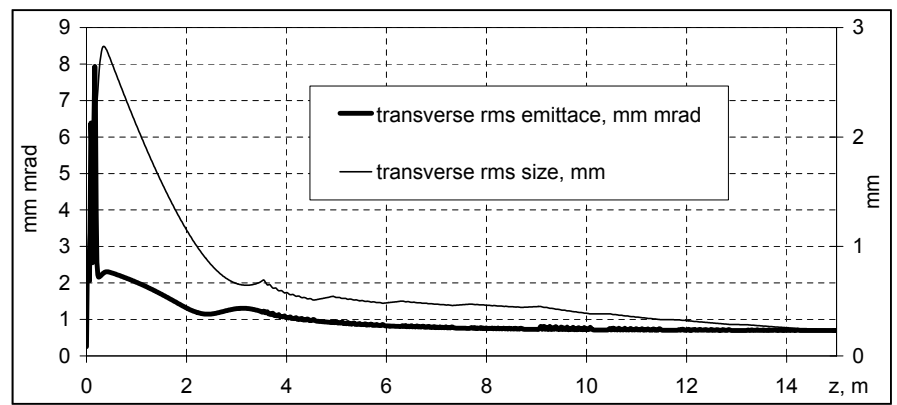

Fig. 5. Beam rms transverse size and projected normalized emittance along the beam line.

\subsection{Transverse and longitudinal phase space}

The characteristic s-shape of the transverse phase space is shown on the left plot in Fig. 8. On the right plot of Fig. 8 the longitudinal phase space of the electron beam at the injector exit $(z=15 \mathrm{~m})$ is presented. It should be noted that the longitudinal 


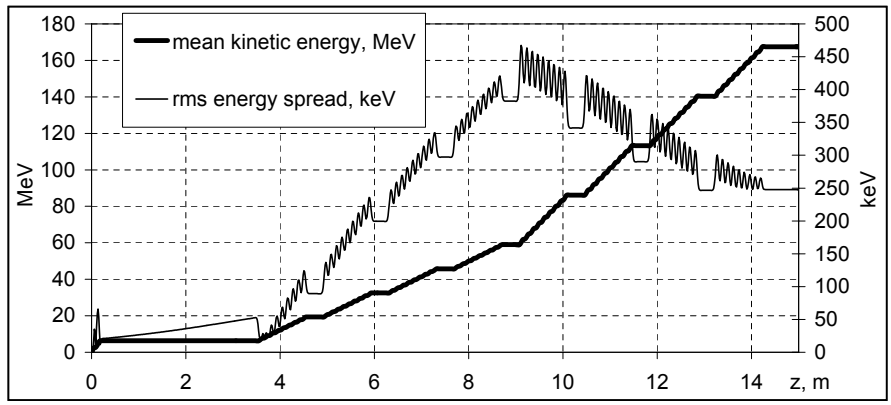

Fig. 6. Beam mean kinetic energy and rms energy spread along the beam line.

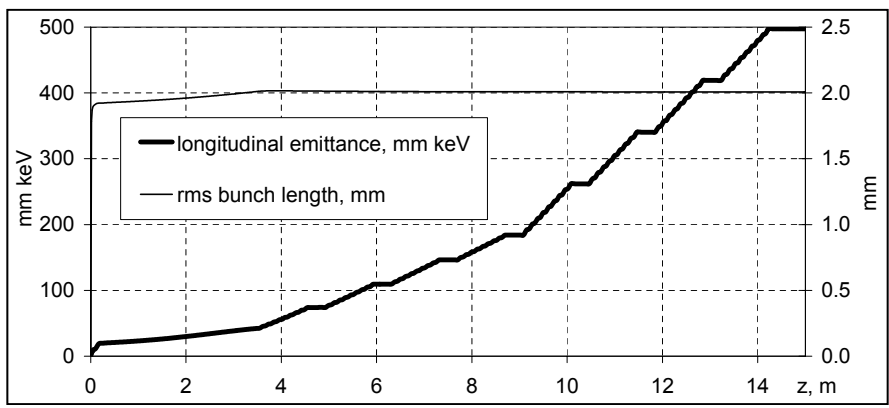

Fig. 7. RMS bunch length and longitudinal emittance along the beam line.

phase space nonlinearity has to be corrected by the third harmonics section downstream the injector. ${ }^{1}$ The third harmonics cavities operated at $3.9 \mathrm{GHz}$ (wavelength $77 \mathrm{~mm}$ ) strongly off-crest in deceleration mode introduce nonlinear correlations in the longitudinal phase space compensating the nonlinearities originating from the injector.

The transverse phase space contains a rather large fraction of peripheric particles, which indeed do not contribute the FEL lasing process. These particles belong mainly to the head and tail, and the local (slice) emittance of these parts of the bunch is rather high (Fig. 9, left plot). The transverse emittance is shown in the right plot of Fig. 9 as a function of the number of core particles taken into consideration for the emittance calculations. Only $1 \%$ charge cut in the phase space results in more than $14 \%$ projected emittance reduction.

\subsection{Influence of key photo injector parameters}

Besides simulations of the optimized setup it is of great importance to study dependences of electron beam properties on machine parameters, which could deviate from the optimized values due to systematic (e.g. precision of set parameter value 

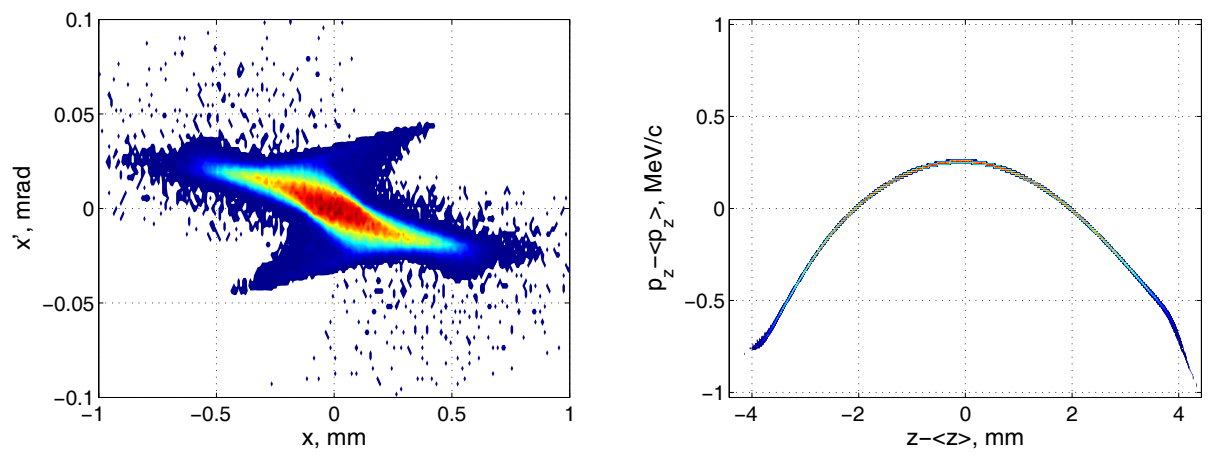

Fig. 8. Transverse (left) and longitudinal (right) phase space of the electron beam at the injector exit $(z=15 \mathrm{~m})$.
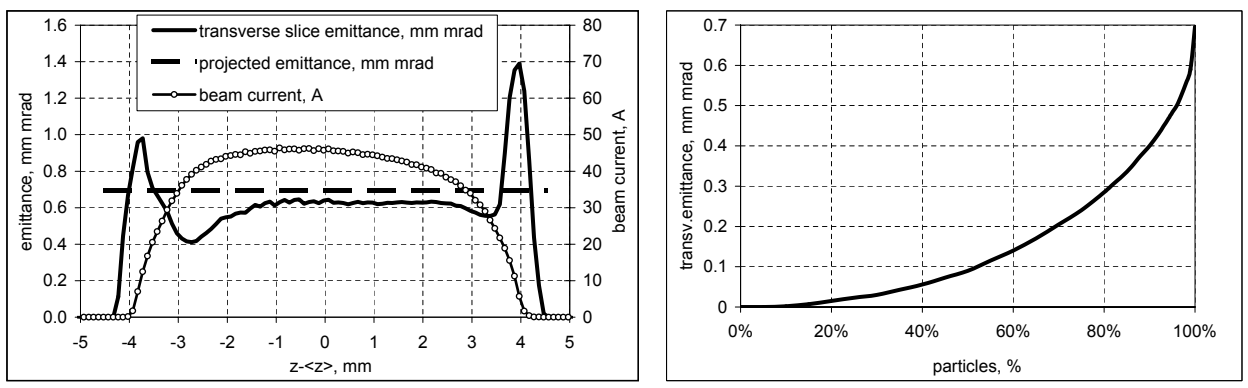

Fig. 9. Left: transverse slice emittance of the electron beam at the injector exit is shown together with projected emittance level and beam current distribution. Right: core emittance-emittance of the beam core vs. number of particles.

to hardware) or stochastic (e.g. jitter) reasons. Another reason to know such dependencies is a normalization procedure of photo injector parameters. A parameters range corresponding to a definite emittance growth (e.g 10\%) is used to obtain a dimensionless variable. This makes search parameter space more homogeneous. Corresponding studies have been performed.

The most sensitive parameter for the emittance of an electron beam is the solenoid peak field. An emittance growth $\frac{\varepsilon-\varepsilon_{\min }}{\varepsilon_{\min }}$ due to the deviation of the main solenoid peak field $B_{z}$ from the optimized value $B_{z}^{o p t}$ (Tab. 1) is shown in Fig. 10 (left). A $0.3 \%$ deviation from the optimum peak field ${ }^{\mathrm{d}}$ results in a $10 \%$ emittance growth. The sensitivity of the electron beam emittance on variation of the cathode laser spot size is illustrated in Fig. 10 (right).

${ }^{\mathrm{d}}$ In terms of the main solenoid current this corresponds to $1.2 \mathrm{~A}$ around the optimum value of 393 A. 

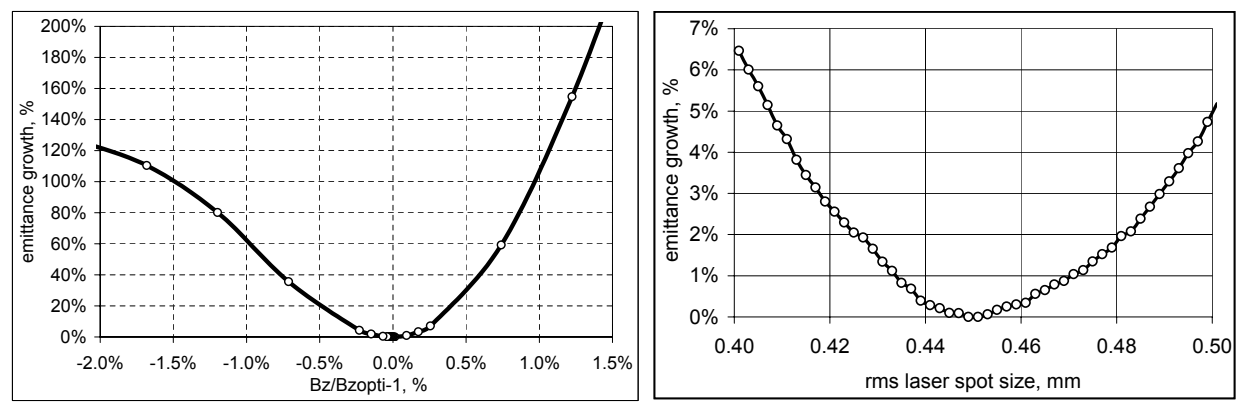

Fig. 10. Emittance growth vs. deviations of injector parameters from the optimized values: main solenoid peak field (left) and rms beam size of the cathode laser (right).
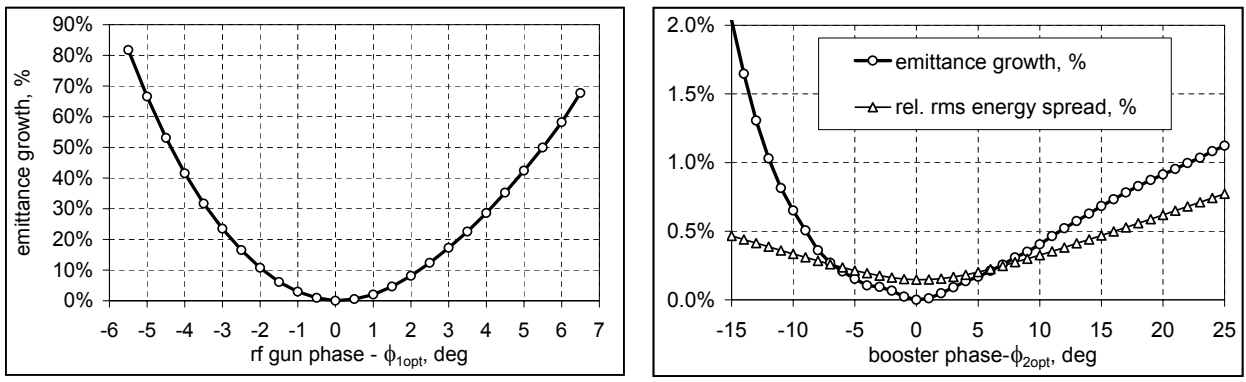

Fig. 11. Left: emittance growth as a function of $\mathrm{rf}$ gun launch phase. Right: emittance growth $\varepsilon / \varepsilon\left(\phi_{2 o p t}\right)-1$ and relative rms energy spread $\sigma_{E} /\langle E\rangle$ as a function of the booster phase.

Another important parameter, whose variation could dilute the quality of the electron beam at the injector exit is the rf gun launch phase. The dependence of the emittance growth due to the variation of the rf gun launch phase is shown in Fig. 11 (left), where the phase is centered to the optimum value $\left(\phi_{1 \text { opt }}=-0.7^{\circ}\right.$ in Tab.1). According to these simulations, a 10\% emittance growth can be caused by an $\mathrm{rf}$ gun phase variation of $\pm 2^{\circ}$. This is much more relaxing than requirements for the phase variation tolerances based on beam energy and bunch charge stability considerations. ${ }^{1}$ An additional charge jitter should be also expected from a charge dependence on rf gun phase (Fig. 1), where the Schottky-like effect results in a non-zero slope $d Q / d \phi_{1}$.

A booster phase scan - the dependence of the emittance growth as a function of the rf phase of the first four cavities in ACC1 - is shown in Fig. 11 on the right plot together with the relative energy spread. It should be noted that tolerances for the energy spread are more stringent, because bunch compression schemes imply rather hard demands on energy correlations within the bunch.

The sensitivity of the beam emittance to the booster gradient is shown in Fig. 12 (left), where emittance growth is shown as a function of the peak electric field in the booster. 

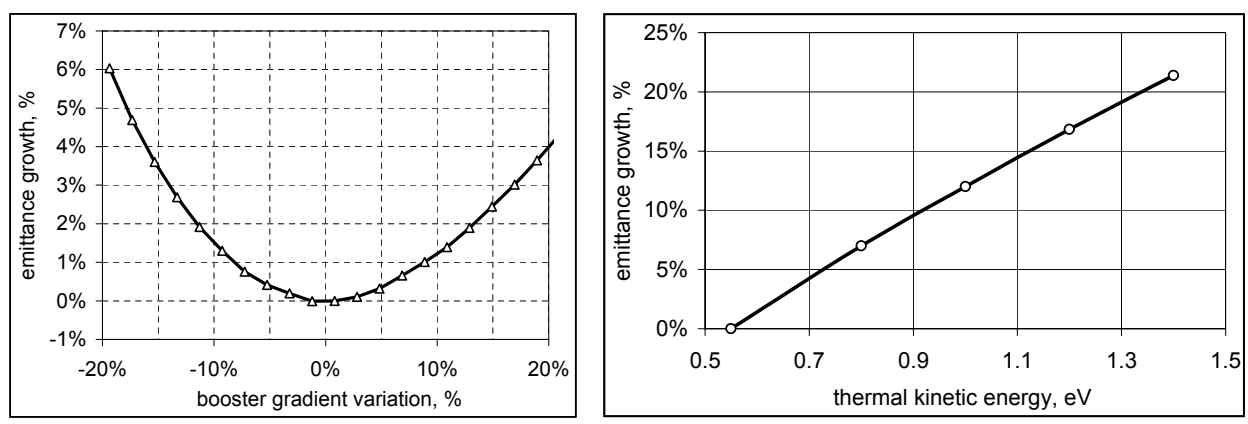

Fig. 12. Left: emittance growth as a function of the booster gradient variation from the optimum value (24.5 MV/m peak field). Right: emittance growth vs. initial kinetic energy of photo electrons. As a reference, the model value $E_{k}=0.55 \mathrm{eV}$ has been used.

The optimized setup for the XFEL photo injector (Tab. 1) assumed the thermal kinetic energy of photo electrons to be $0.55 \mathrm{eV}$. This corresponds to the theoretical model for $C s_{2} \mathrm{Te}$ photo cathodes. ${ }^{9}$ The projected transverse emittance for this case $(0.7 \mathrm{~mm} \mathrm{mrad})$ contains more than $50 \%$ thermal emittance $(0.38 \mathrm{~mm} \mathrm{mrad})$, which is uncorrelated and cannot be compensated. Recent measurements performed at PITZ $^{10}$ yielded initial kinetic energies of $\sim 1.4 \mathrm{eV}$. In order to study the influence of the thermal emittance on the final projected emittance of the electron beam at the injector exit, corresponding simulations have been performed. Emittance growth as a function of the initial kinetic energy of photo electrons is shown in Fig. 12 (right). From this plot the increase of the initial thermal kinetic energy close to the measured one $(1.4 \mathrm{eV})$ results in more than $20 \%$ increase of the final emittance.

The cathode laser temporal profile plays a significant role in a formation of the electron beam projected emittance. A flat-top profile with 20 ps FWHM has been used. The rise and fall time is responsible for the contribution to the projected emittance from head and tail of the bunch (Fig. 9). The dependence of the emittance growth due to the variation of the rise and fall time is shown in Fig. 13(left). As a reference, a projected emittance for the cathode laser with 2 ps has been used. Typical temporal laser profiles used for the simulations are shown in the right plot of Fig. 13.

\section{Conclusions}

The beam dynamics optimization procedure for the European XFEL photo injector has been developed using a staged strategy, including photo emission, rf gun and booster parameter tuning. Application of this approach based on ASTRA simulations resulted in an optimized photo injector setup delivering a projected normalized transverse emittance of $0.7 \mathrm{~mm}$ mrad for $1 \mathrm{nC}$ beam. The influence of photo injector key parameters has been studied; the most sensitive parameters are main solenoid 

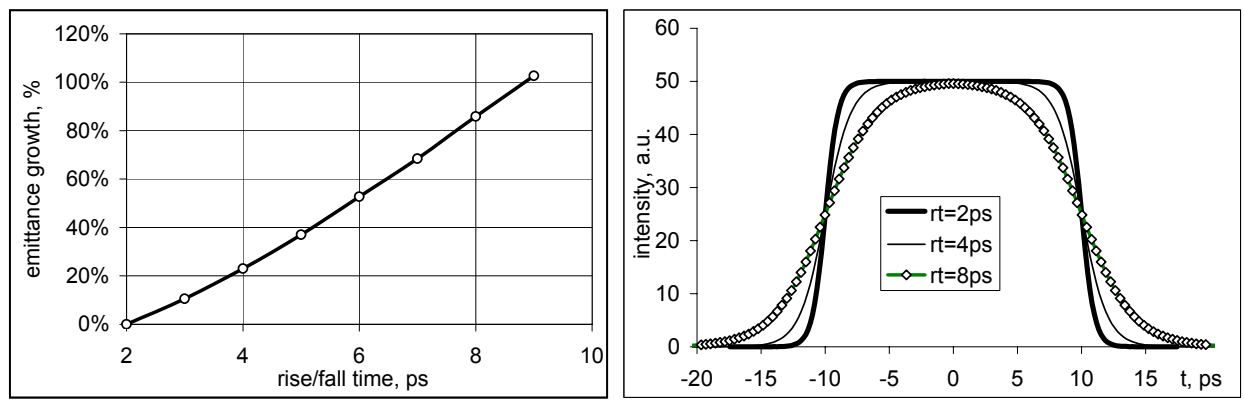

Fig. 13. Emittance growth vs. rise/fall time of the cathode laser. Examples of temporal laser profiles used for the simulations (right plot), rise and fall time were varied whereas the length (FWHM) was fixed to $20 \mathrm{ps.}$

strength, rise and fall time of the cathode laser temporal profile and initial kinetic energy of the photo electrons.

\section{Acknowledgments}

The author thanks F. Stephan, K. Floettmann, C. Boulware and the PITZ group members for useful discussions.

\section{References}

1. M. Altarelli et al, The European X-Ray Free Electron Laser, DESY 2006-097 (2007).

2. B.E. Carlsten, Space Charge Induced Emittance Compensation in High Brightness Photoinjectors, Part. Accel 49 (1995) p27-65.

3. L. Serafini and J. B. Rosenzweig, Phys. Rev. E 55, 7565 (1997).

4. K.Floettmann, A Space charge Tracking Algorithm (ASTRA), user manual, http://www.desy.de/ mpyflo/Astra_dokumentation/.

5. G. Suberlucq, Development and production of photo cathodes for the CLIC test facility, in Proc. of 18th International FEL conference, Rome, Italy, 1996, p. II-131.

6. M.Krasilnikov et al, NIMA, 558, 249-252 (2006).

7. M. Krasilnikov et al., Recent developments at PITZ, in Proc. PAC'05 USA, Knoxwille, May 2005, p. 1012.

8. S.Rimjaem et al., Tuning and Conditioning of a New High Gradient Gun Cavity at PITZ, in Proc. EPAC'08, Italy, Genoa

9. K.Floettmann Note on the thermal emittance of electrons emitted by Cesium Telluride photo cathodes, TESLA FEL Reports 1997-01, 1997.

10. S.Lederer et al., Investigations on the Thermal Emittance of Cs2Te Photocathodes at PITZ, in Proc. FEL 200\%, Russia, Novosibirsk, p.350.

11. N.Metropolis and S.Ulam, The Monte Carlo Method, J. Amer. Stat. Assoc., 44, 335341 (1949).

12. J.A. Nelder and R. Mead, Computer Journal, 7, 308-313 (1965). 